TITLE:

\title{
Huge electron-hole exchange interaction in aluminum nitride
}

$\operatorname{AUTHOR}(S)$ :

Ishii, Ryota; Funato, Mitsuru; Kawakami, Yoichi

CITATION:

Ishii, Ryota ...[et al]. Huge electron-hole exchange interaction in

aluminum nitride. Physical Review B 2013, 87(16): 161204.

ISSUE DATE:

2013-04

URL:

http://hdl.handle.net/2433/174057

RIGHT:

C)2013 American Physical Society 


\title{
PHYSICAL REVIEW B 87, 161204(R) (2013)
}

\section{gु \\ Huge electron-hole exchange interaction in aluminum nitride}

\author{
Ryota Ishii, ${ }^{*}$ Mitsuru Funato, and Yoichi Kawakami ${ }^{\dagger}$ \\ Department of Electronic Science and Engineering, Kyoto University, Kyoto 615-8510, Japan
}

(Received 20 March 2013; published 30 April 2013)

\begin{abstract}
Optical spectroscopy is performed for $c$-plane homoepitaxial aluminum nitride (AlN) films. The temperature dependence of the polarization-resolved photoluminescence spectra reveals the exciton fine structure. The experimental results demonstrate that the electron-hole exchange interaction energy $(j)$ in $\mathrm{AlN}$ is $j=6.8 \mathrm{meV}$, which is the largest value for typical III-V and II-VI compound semiconductors. We propose the effective interatomic distance as the criterion of the electron-hole exchange interaction energy, revealing a universal rule. This study should encourage potential applications of excitonic optoelectronic devices in nitride semiconductors similar to those using II-VI compound semiconductors.
\end{abstract}

DOI: 10.1103/PhysRevB.87.161204

PACS number(s): 71.70.Gm, 71.35.Cc, 71.55.Eq

Excitons, which are a type of fundamental excitation in crystals, dominate optical transitions in semiconducting materials at low temperatures. ${ }^{1,2}$ In wide band-gap materials, such as oxide or nitride semiconductors, an exciton may exist up to room temperature due to its small Bohr radius. Consequently, excitonic effects have been extensively studied in oxide semiconductors toward realizing, for example, low-threshold lasing. ${ }^{3,4}$ However, in nitride semiconductors, the impact of excitonic effects on the performance of optoelectronic devices has not been considered explicitly. ${ }^{5,6}$ This is partly because the relatively poor crystalline quality hinders the intrinsic exciton physics, and essentially because the excitonic correlations in $\mathrm{GaN}$ are weak compared with those in $\mathrm{ZnO}$. One gauge of the excitonic interaction is the electron-hole exchange interaction energy $j$, which is estimated to be $1.2 \mathrm{meV}^{7}$ and $5.8 \mathrm{meV}^{8}$ in $\mathrm{GaN}$ and $\mathrm{ZnO}$, respectively. This is why an excitonic nature has yet to be observed in current GaN-based optoelectronic devices, such as blue light-emitting diodes ${ }^{9}$ or green laser diodes. ${ }^{10}$

Recently, aluminum nitride (AlN) has realized a new frontier of nitride semiconductor optoelectronic technology into the deep ultraviolet spectral region due to its distinguished nature. (The band gap $E_{g}$ is $6.0 \mathrm{eV}$.) ${ }^{11}$ The exciton Bohr radius and the exciton binding energy of AlN are $\sim 1.5 \mathrm{~nm}$ and $\sim 53 \mathrm{meV}$, respectively. These values are comparable to those of $\mathrm{ZnO}$. Consequently, huge excitonic correlations should exist in AlN. To date, the electron-hole exchange interaction in AlN has been studied in only one work and is estimated to be $j=-4.0 \mathrm{meV} .{ }^{12}$ However, if the sign of $j$ is negative, a spin-triplet exciton is energetically higher than a spin-singlet exciton, which contradicts the results of both solid state physics within a framework of a simple two-band approximation ${ }^{13}$ and previous experiments on any other semiconducting materials. Therefore, the electron-hole exchange interaction in AlN should be examined in detail.

In this study, photoluminescence (PL) spectroscopy is performed on state-of-the-art $c$-plane homoepitaxial AlN films. The temperature dependence of the polarization-resolved PL spectra reveals the exciton fine structure. The experimental results indicate that in AlN the electron-hole exchange interaction energy $j$ is $6.8 \mathrm{meV}$, which is the largest value among typical III-V and II-VI compound semiconductors. We propose that as a universal rule, the effective interatomic distance is a criterion of $j$.
Homoepitaxial AlN films are grown by metal-organic vapor phase epitaxy on the $c$-plane AlN bulk substrates prepared by a physical vapor transport method. The $\mathrm{x}$-ray rocking curves of (0002) symmetric and (1102) asymmetric planes for the epilayer are 14 and 29 arcsec, respectively. The carbon concentration in the epilayer is below the detection limit of secondary ion mass spectroscopy, which is $<3 \times 10^{17} \mathrm{~cm}^{-3}$. The oxygen and the silicon concentrations are near the detection limit of $6 \times 10^{17} \mathrm{~cm}^{-3}$ and $1 \times 10^{16} \mathrm{~cm}^{-3}$, respectively. The results indicate that homoepitaxial AIN films have excellent crystalline qualities. Pretreatment of the substrate and growth conditions are detailed elsewhere. ${ }^{14}$

PL spectroscopy is performed for the $c$-plane homoepitaxial AlN films using a pulsed $\operatorname{ArF}$ excimer laser $(\lambda=193 \mathrm{~nm})$. The repetition rate and the pulse width are $25 \mathrm{~Hz}$ and $4 \mathrm{~ns}$, respectively. The PL signals are dispersed by a $50 \mathrm{~cm}$ monochromator with a $\mathrm{LN}_{2}$-cooled charge-coupled device. The spectral resolution is about $0.045 \mathrm{~nm}$ at a wavelength of $200 \mathrm{~nm}$, which corresponds to about $1 \mathrm{meV}$. The temperature dependence is measured using a closed-cycle cryostat from 10 to $300 \mathrm{~K}$. Herein, we carry out PL spectroscopy under two experimental configurations. In Config. (a), the sample is excited from the surface normal, and the PL signal is detected at an angle of $60^{\circ}$ with the surface normal. In Config. (b), the PL signal of a sample is detected from the surface normal, and the sample is excited at an angle of $60^{\circ}$ with the surface normal.

Before describing the experimental results, the electronic band structure of AlN should be recalled. The conduction and valence band edges are assumed to be composed of the atomic $s$ orbital and $p$ orbital, respectively. The $1 s$ excitonic effective Hamiltonian in wurtzite crystals is derived by the theory of invariants as ${ }^{15}$

$$
\begin{aligned}
H= & \Delta_{1} J_{z}^{2}+\Delta_{2} J_{z} \sigma_{v z}+\Delta_{3}\left(\sigma_{+v} J_{-}+\sigma_{-v} J_{+}\right) \\
& +\frac{1}{2} j \sigma_{e} \sigma_{h},
\end{aligned}
$$

where $\Delta_{1}$ is the crystal-field split interaction energy, and $\Delta_{2,3}$ are the parallel $(\| c)$ and perpendicular $(\perp c)$ components of the spin-orbit interaction energy. $j$ is an electron-hole exchange interaction energy, which is the subject in this paper. The other notations in Eq. (1) are the same as those in Ref. 7. Numerous works have investigated $\Delta_{1-3}$ in AlN. These studies have revealed a negatively large $\Delta_{1}$ and a positively small 


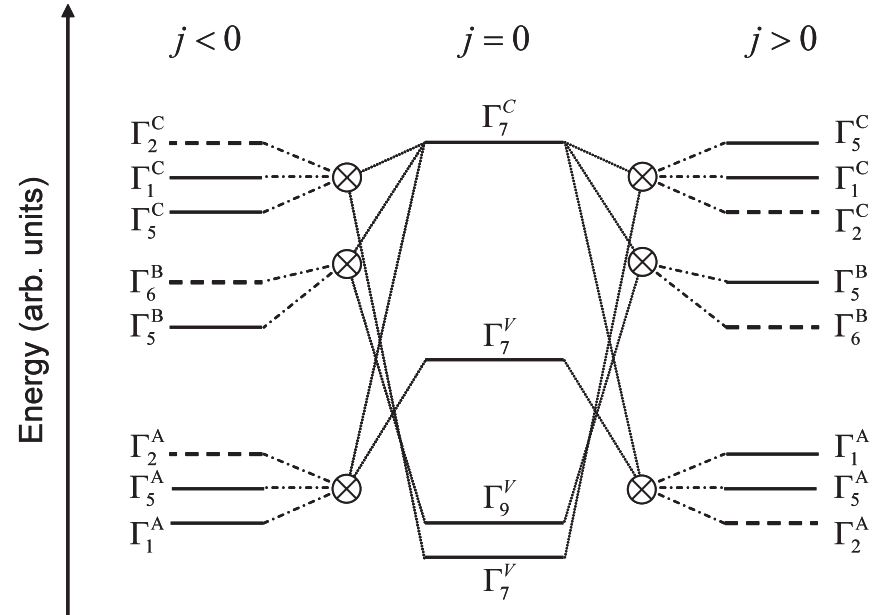

FIG. 1. Electronic band or exciton fine structure of AlN, $j<0$ (left), $j=0$ (center), and $j>0$ (right). $\Gamma_{1}$ and $\Gamma_{5}$ are dipole-allowed states (solid lines) for $E \| c$ and $E \perp c$, respectively. $\Gamma_{2}$ and $\Gamma_{6}$ are dipole-forbidden states (broken lines).

$\Delta_{2,3} \cdot{ }^{16-18}$ The center diagram in Fig. 1 shows the electronic band structure of AIN. From low to high electron energy, the valence band ordering in $\mathrm{AlN}$ is $\Gamma_{7}^{v}-\Gamma_{9}^{v}-\Gamma_{7}^{v}$.

As already mentioned, a negative electron-hole exchange interaction energy in AIN has been reported. ${ }^{12}$ If the sign of $j$ is negative, the exciton fine structure of AIN is represented as the left diagram in Fig. 1. On the other hand, if the sign of $j$ is positive, the exciton fine structure is represented as the right diagram in Fig. 1. The oscillator strength of $\Gamma_{5}^{\mathrm{A}}$ is much smaller than that of $\Gamma_{1}^{\mathrm{A}}$ in both the cases. Figure 1 suggests that the sign of $j$ can be estimated by the energy ordering of $\Gamma_{1}^{\mathrm{A}}$ and $\Gamma_{5}^{\mathrm{A}}$.

Figure 2 shows a typical PL spectrum for our $c$-plane homoepitaxial AIN films taken under a weak excitation condition at $10 \mathrm{~K}$ with the experimental geometry of Config. (a). Several sharp peaks are observed, which are called $\mathrm{D}^{0} \mathrm{X}$, $\mathrm{X}, \alpha$, and FX, in this paper. Neuschl et al. suggested that the

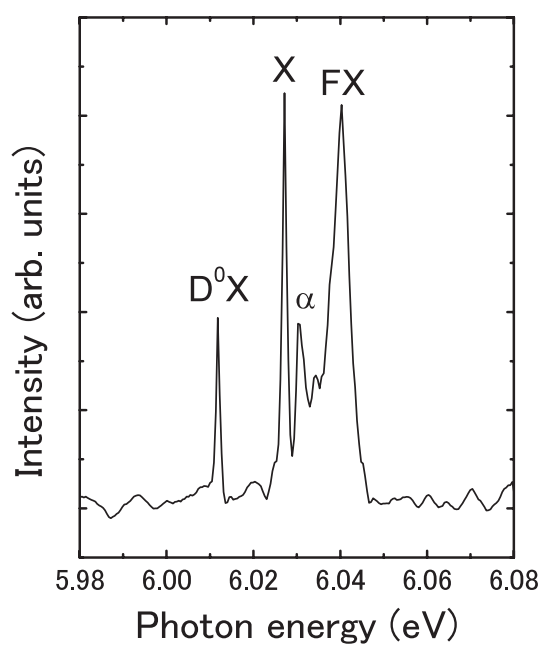

FIG. 2. Typical PL spectrum of our $c$-plane homoepitaxial AIN films taken under the excitation power density of $6 \mathrm{~kW} / \mathrm{cm}^{2}$ at $10 \mathrm{~K}$. Experimental geometry is Config. (a).
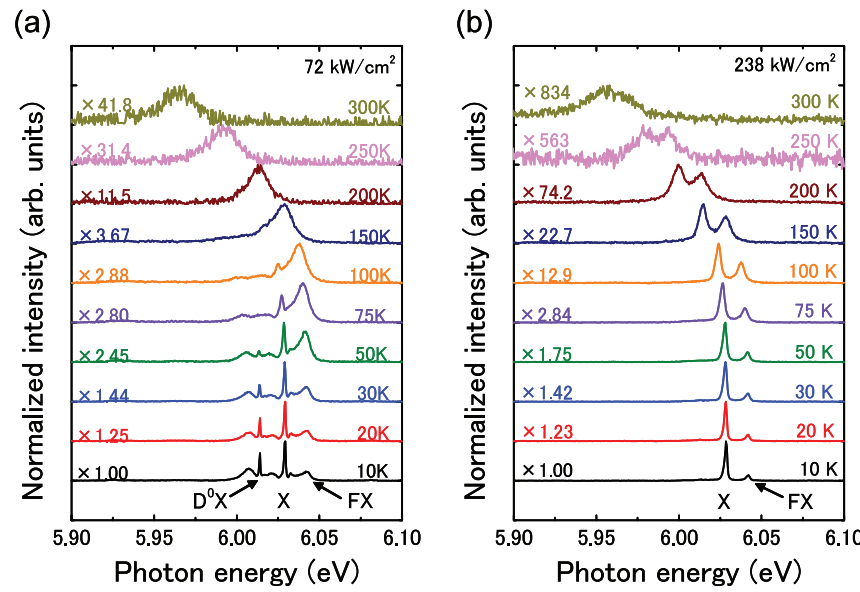

FIG. 3. (Color online) Temperature-dependent PL spectra for the $c$-plane homoepitaxial film (a) under Config. (a) and (b) under Config. (b). Respective excitation power densities are $72 \mathrm{~kW} / \mathrm{cm}^{2}$ and $238 \mathrm{~kW} / \mathrm{cm}^{2}$. At only the PL spectrum at $300 \mathrm{~K}$ in Fig. 3(b), excitation power density is $714 \mathrm{~kW} / \mathrm{cm}^{2}$ due to the low $\mathrm{S} / \mathrm{N}$ ratio and the spectrum is divided by 3 .

origin of the $\mathrm{D}^{0} \mathrm{X}$ peak is a silicon donor-bound exciton. ${ }^{19}$ The $\alpha$ peak has not been identified. The thermal behavior of the FX peak located at a higher energy unambiguously indicates the free excitonic nature. Although the $\mathrm{X}$ peak has been assigned to the bound state of impurities, this assignment remains debatable due to polarization properties. Our previous work demonstrated that the luminescence peaks of $\mathrm{D}^{0} \mathrm{X}$ and FX are polarized parallel to the $c$ axis $(E \| c)$ while the luminescence peak of $\mathrm{X}$ is polarized perpendicular to the $c$ axis $(E \perp c) .{ }^{14}$ The origin of the FX peak is identified as $\Gamma_{1}^{\mathrm{A}}$, but a simple shallow bound exciton picture cannot account for the observed polarization of the $\mathrm{X}$ peak.

In this paper, we identify the origin of the $\mathrm{X}$ peak by the temperature dependence of the polarization-resolved PL spectra for $c$-plane homoepitaxial AlN films. Figures 3(a) and 3(b) show the temperature-dependent PL spectra with the experimental geometry of Config. (a) and (b), respectively. The PL spectra are rather different from that of Fig. 2 due to the excitation power density. In Fig. 3(a), the free excitonic nature of the FX peak is clearly observed, while in Fig. 3(b), the luminescence of the $X$ peak is observed up to room temperature despite the $13.6 \mathrm{meV}$ energy difference between the $\mathrm{X}$ and FX peaks. The $\mathrm{X}$ peak does not seem to be a bound state of the FX peak. Hence, the temperature dependence of the PL integrated intensity is quantitatively estimated for each peak by the following equation:

$$
I(T)=I(0) /\left\{1+A \exp \left(-E_{a} / k_{B} T\right)\right\},
$$

where $E_{a}$ and $k_{B}$ are the thermal activation energy and Boltzmann constant, respectively. $A$ characterizes the oscillator strength of a peak. The thermal activation energies of $\mathrm{D}^{0} \mathrm{X}$, $\mathrm{X}$, and FX are estimated to be $6.4 \mathrm{meV}, 42 \mathrm{meV}$, and $46 \mathrm{meV}$, respectively (Fig. 4). The activation energy of the $\mathrm{X}$ peak is much larger than the energy difference between the peaks of $\mathrm{X}$ and FX (13.6 meV). Furthermore, the activation energy is nearly the same as the free exciton binding energy derived from the effective mass approximation. ${ }^{14}$ Therefore, we conclude 


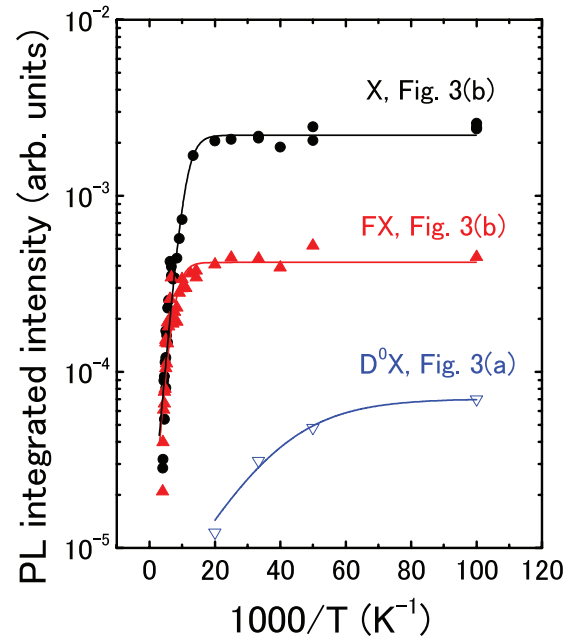

FIG. 4. (Color online) Arrhenius plots of each peak, $\mathrm{D}^{0} \mathrm{X}, \mathrm{X}$, and FX.

that the $\mathrm{X}$ peak originates from the free exciton with an irreducible representation of $\Gamma_{5}^{\mathrm{A}}$. This interpretation can also readily explain the aforementioned optical polarization property of the X peak $(E \perp c)$. The energy ordering of $\Gamma_{5}^{\mathrm{A}}$ and $\Gamma_{1}^{\mathrm{A}}$ indicates that the electron-hole exchange interaction energy in AlN has to be positive (right diagram in Fig. 1), and is estimated to be $j=6.8 \mathrm{meV}$ by Eq. (1). The larger thermal quenching of the $\mathrm{X}$ peak is consistent with the theoretical prediction of a much smaller oscillator strength. It should be noted that our interpretation is also consistent with the results of reflectance spectroscopy for AlN bulk substrates under uniaxial stress. ${ }^{18}$

The origin of the $\alpha$ peak should be discussed considering the aforementioned results. The donor binding energy in AlN is estimated to be $\sim 60 \mathrm{meV}$ via the effective mass theory. Applying the Hayne's rule of GaN to AlN, the binding energy of a donor bound exciton in $\mathrm{AlN}$ is $\sim 12 \mathrm{meV}$. This value is nearly the same as the energy difference between the peaks of FX and $\alpha(9.5 \mathrm{meV})$. Therefore, we suggest that the $\alpha$ peak originates from the donor bound exciton of the FX peak.

Above, the electron-hole exchange interaction is experimentally studied in AlN. Below, we discuss the value of $j$ in typical III-V and II-VI compound semiconductors.

Rohner showed that $j$ is about $10-20 \%$ of the exciton binding energy in II-VI compound semiconductors and is correlated with $\epsilon /(\mu a),{ }^{20}$ where $\epsilon, \mu$, and $a$ are the dielectric constant, the exciton reduced-mass, and the lattice constant, respectively. Wardzyński et al. demonstrated that $j$ is correlated with the interatomic distance. ${ }^{21}$ Julier et al. expanded the interpretation to $\mathrm{GaN} .{ }^{22} \mathrm{Fu}$ et al. suggested that $j$ is correlated with the exciton Bohr radius. ${ }^{23}$ However, the methods proposed in Refs. 20 and 23 are not applicable to anisotropic materials and uncertainties about the choice of hole mass remain. The method proposed in Refs. 21 and 22 can explain the behavior only in common-anion (cation) materials. Therefore, another gauge that can universally explain the value of $j$ in any semiconducting materials should be established.

Here, we treat the exchange interaction problem between an electron and a hole under the Hartree-Fock approximation. The effective mass approximation is assumed to describe the contributions from other particles. Subsequently, the Coulomb interaction can be expressed by the potential as

$$
V=\frac{q_{1} q_{2}}{4 \pi} \frac{1}{\sqrt{\epsilon_{2} \epsilon_{3} x^{2}+\epsilon_{3} \epsilon_{1} y^{2}+\epsilon_{1} \epsilon_{2} z^{2}}},
$$

where $q_{1,2}$ are electric charges and $\left(\epsilon_{1}, \epsilon_{2}, \epsilon_{3}\right)$ is the dielectric tensor in $x y z$ cartesian coordinates. $\epsilon_{1}=\epsilon_{2}=\epsilon_{3}=\epsilon$ holds in zincblende (ZB) semiconductors, while $\epsilon_{1}=\epsilon_{2}=\epsilon_{\perp}$ and $\epsilon_{3}=\epsilon_{\|}$hold in wurtzite (WZ) semiconductors. The electronhole exchange interaction should be related to $V$, and only the dielectric constant and distance between the charges are material-dependent terms. The latter can be characterized by interatomic distance $d$ as shown in Ref. 21. $d=(\sqrt{3} / 4) a$

TABLE I. Material parameters in typical III-V and II-VI compound semiconductors. Unless stated, those in nitride semiconductors are picked up from Ref. 25, while in others are picked up from Ref. 26.

\begin{tabular}{|c|c|c|c|c|c|c|c|c|}
\hline Materials & $E_{g}(\mathrm{eV})$ & $a(\AA)$ & $c(\AA)$ & $u$ & $\epsilon$ & $\epsilon_{\perp}$ & $\epsilon_{\|}$ & $j(\mathrm{meV})$ \\
\hline $\mathrm{ZnS}(\mathrm{ZB})$ & 3.84 & 5.4053 & & & 8.3 & & & 4.0 (Ref. 8) \\
\hline $\mathrm{ZnSe}(\mathrm{ZB})$ & 2.82 & 5.6674 & & & 8.6 & & & 1.0 (Ref. 8) \\
\hline $\mathrm{ZnTe}(\mathrm{ZB})$ & 2.395 & 6.0882 & & & 10.3 & & & 0.28 (Ref. 21) \\
\hline $\mathrm{GaP}(\mathrm{ZB})$ & 2.350 & 5.45064 & & & 11.1 & & & 0.175 (Ref. 27) \\
\hline $\mathrm{CdTe}(\mathrm{ZB})$ & 1.606 & 6.46 & & & 10.4 & & & 0.07 (Ref. 28) \\
\hline GaAs (ZB) & 1.519 & 5.65359 & & & 12.8 & & & $\begin{array}{c}0.015 \text { (Ref. 29) } \\
0.075 \text { (Ref. 30) }\end{array}$ \\
\hline InP (ZB) & 1.424 & 5.8687 & & & 12.6 & & & 0.04 (Ref. 29) \\
\hline AlN (WZ) & 6.10 & 3.112 & 4.982 & 0.3819 (Ref. 34) & & 7.8 (Ref. 35) & 9.3 (Ref. 35) & 6.8 (This work) \\
\hline $\mathrm{GaN}(\mathrm{WZ})$ & 3.51 & 3.189 & 5.145 & 0.3772 (Ref. 34) & & 9.3 (Ref. 36) & 10.1 (Ref. 36) & 1.2 (Ref. 7) \\
\hline $\mathrm{ZnO}(\mathrm{WZ})$ & 3.44 & 3.2496 & 5.2042 & 0.3807 (Ref. 34) & & 7.8 & 8.8 & $\begin{array}{l}5.6 \text { (Ref. 8), } \\
4.9 \text { (Ref. 31), } \\
4.73 \text { (Ref. 32) }\end{array}$ \\
\hline CdS (WZ) & 2.58 & 4.1348 & 6.7490 & 0.3773 (Ref. 37) & & 8.3 & 8.7 & 2.5 (Ref. 8) \\
\hline CdSe (WZ) & 1.84 & 4.2999 & 7.0109 & 0.3767 (Ref. 37) & & 9.3 & 10.2 & $\begin{array}{c}0.13 \text { (Ref. 33), } \\
0.4 \text { (Ref. 8) }\end{array}$ \\
\hline
\end{tabular}




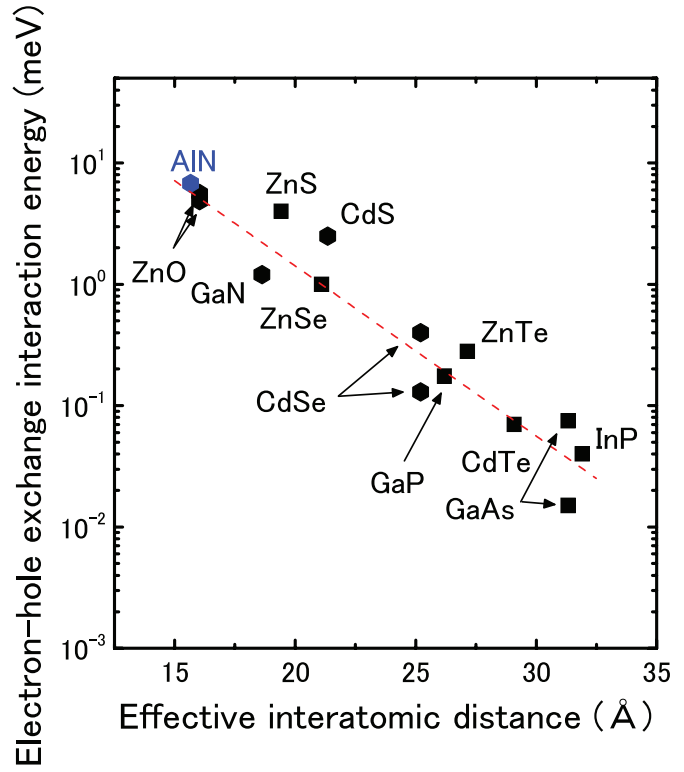

FIG. 5. (Color online) Effective interatomic distance vs the electron-hole exchange interaction energy in typical III-V and II-VI compound semiconductors.

holds in zincblende semiconductors, while $d_{\|}=u c$ and $d_{\perp}=\sqrt{a^{2} / 3+(1 / 2-u)^{2} c^{2}}$ hold in wurtzite semiconductors, where $c$ and $u$ are the $c$-axis lattice constant and an internal parameter, respectively. ${ }^{24}$ Therefore, we propose an effective interatomic distance $d_{\text {eff }}$ as a gauge of the electron-hole exchange interaction energy as

$$
\begin{aligned}
d_{\mathrm{eff}}^{\mathrm{ZB}} & =\epsilon \times d, \\
d_{\mathrm{eff}}^{\mathrm{WZ}} & =\sqrt{\left(2 \epsilon_{\perp} \epsilon_{\|} d_{\perp}^{2}+\epsilon_{\perp}^{2} d_{\|}^{2}\right) / 3 .}
\end{aligned}
$$

Table I summarizes the material parameters in typical III-V and II-VI compound semiconductors. Figure 5 shows the relation between $d_{\mathrm{eff}}$ and $j$. The value of $j$ can be characterized by $d_{\mathrm{eff}}$, which strongly supports the result in this paper of $j=6.8 \mathrm{meV}$ in AIN.

In conclusion, the electron-hole exchange interaction energy is experimentally demonstrated to be $j=6.8 \mathrm{meV}$, which is the largest value among typical III-V and II-VI compound semiconductors. It should be noted that $j$ is a crucial parameter to understand the excitonic properties in wide band-gap materials, and for example, is related to an open problem on the valence band ordering in $\mathrm{ZnO} .^{38,39} \mathrm{As}$ a gauge of the electron-hole exchange interaction energy, the effective interatomic distance $d_{\text {eff }}$ is proposed.

The authors would like to thank K. Matsuda and R. G. Banal for fabricating the crystals. This research was partially supported by a Grant-in-Aid for the Japan Society for the Promotion of Science as well as the Mizuho Foundation for the Promotion of Sciences. *ryota.ishii@optomater.kuee.kyoto-u.ac.jp

${ }^{\dagger}$ kawakami@kuee.kyoto-u.ac.jp

${ }^{1}$ R. S. Knox, Theory of excitons (Academic Press, New York, 1963).

${ }^{2}$ D. C. Reynolds and T. C. Collins, Excitons, their properties and uses (Academic Press, New York, 1981).

${ }^{3}$ D. M. Bagnall, Y. F. Chen, Z. Zhu, T. Yao, S. Koyama, M. Y. Shen, and T. Goto, Appl. Phys. Lett. 70, 2230 (1997).

${ }^{4}$ C. Klingshirn, R. Hauschild, J. Fallert, and H. Kalt, Phys. Rev. B 75, 115203 (2007).

${ }^{5}$ J. Piprek, Nitride Semiconductor Devices: Principles and Simulations (Wiley-VCH, New York, 2007).

${ }^{6} \mathrm{H}$. Morkoç, Handbook of Nitride Semiconductors and Devices (Wiley-VCH, Weinheim, 2008).

${ }^{7}$ R. Ishii, A. Kaneta, M. Funato, Y. Kawakami, and A. A. Yamaguchi, Phys. Rev. B 81, 155202 (2010).

${ }^{8}$ D. W. Langer, R. N. Euwema, K. Era, and T. Koda, Phys. Rev. B 2 , 4005 (1970).

${ }^{9}$ S. Nakamura, T. Mukai, and M. Senoh, Appl. Phys. Lett. 64, 1687 (1994).

${ }^{10}$ Y. Enya, Y. Yoshizumi, T. Kyono, K. Akita, M. Ueno, M. Adachi, T. Sumitomo, S. Tokuyama, T. Ikegami, K. Katayama, and T. Nakamura, Appl. Phys. Express 2, 082101 (2009).

${ }^{11}$ Y. Taniyasu, M. Kasu, and T. Makimoto, Nature (London) 441, 325 (2006).

${ }^{12}$ M. Feneberg, M. Romero, B. Neuschl, K. Thonke, M. Röppischer, C. Cobet, N. Esser, M. Bickermann, and R. Goldhahn, Appl. Phys. Lett. 102, 052112 (2013).

${ }^{13}$ G. Grosso and G. P. Parravicini, Solid State Physics (Academic Press, London, 2000).
${ }^{14}$ M. Funato, K. Matsuda, R. G. Banal, R. Ishii, and Y. Kawakami, Appl. Phys. Express 5, 082001 (2012).

${ }^{15}$ K. Cho, Phys. Rev. B 14, 4463 (1976).

${ }^{16}$ J. Chen, W. Z. Shen, H. Ogawa, and Q. X. Guo, Appl. Phys. Lett. 85, 4334 (2004).

${ }^{17}$ E. Silveira, J. A. Freitas, Jr., O. J. Glembocki, G. A. Slack, and L. J. Schowalter, Phys. Rev. B 71, 041201(R) (2005).

${ }^{18}$ R. Ishii, A. Kaneta, M. Funato, and Y. Kawakami (unpublished).

${ }^{19}$ B. Neuschl, K. Thonke, M. Feneberg, S. Mita, J. Xie, R. Dalmau, R. Collazo, and Z. Sitar, Phys. Status Solidi B 249, 511 (2012).

${ }^{20}$ P. G. Rohner, Phys. Rev. B 3, 433 (1971).

${ }^{21}$ W. Wardzyński and M. Suffczyński, Solid State Commun. 10, 417 (1972).

${ }^{22}$ M. Julier, J. Campo, B. Gil, J. P. Lascaray, and S. Nakamura, Phys. Rev. B 57, R6791 (1998).

${ }^{23}$ H. Fu, L. W. Wang, and A. Zunger, Phys. Rev. B 59, 5568 (1999).

${ }^{24} d_{\perp}$ is assumed to characterize the physics perpendicular to the $c$ axis.

${ }^{25}$ I. Vurgaftman and J. R. Meyer, J. Appl. Phys. 94, 3675 (2003).

${ }^{26}$ O. Madelung, Semiconductors: Data Handbook 3rd edition (Springer, Berlin, 2004)

${ }^{27}$ Y. Yafet and T. Thomas, Phys. Rev. 131, 2405 (1963).

${ }^{28}$ M. Suffczyński and W. Wardzyński, Phys. Lett. A 36, 29 (1971).

${ }^{29}$ W. Ekardt, K. Lösch, and D. Bimberg, Phys. Rev. B 20, 3303 (1979).

${ }^{30}$ D. D. Sell, S. E. Stokowski, R. Dingle, and J. V. DiLorenzo, Phys. Rev. B 7, 4568 (1973)

${ }^{31}$ J. Wrzesinski and D. Fröhlich, Phys. Rev. B 56, 13087 (1997). 
${ }^{32}$ B. Gil, A. Lusson, V. Sallet, S. Hassani, R. Triboulet, and P. Bigenwald, Jpn. J. Appl. Phys. 40, L1089 (2001).

${ }^{33}$ V. P. Kochereshko, G. V. Mikhailov, and I. N. Ural'tsev, Fiz. Tverd. Tela 25, 769 (1983) [Sov. Phys. Solid State 25, 439 (1983)].

${ }^{34}$ Q. Yan, P. Rinke, M. Winkelnkemper, A. Qteish, D. Bimberg, M. Scheffler, and C. G. Van de Walle, Semicond. Sci. Technol. 26, 014037 (2011).

${ }^{35}$ W. J. Moore, J. A. Freitas, R. T. Holm, O. Kovalenkov, and V. Dmitriev, Appl. Phys. Lett. 86, 141912 (2005).
${ }^{36}$ T. Azuhata, T. Sota, K. Suzuki, and S. Nakamura, J. Phys.: Condens. Matter 7, L129 (1995).

${ }^{37}$ H. Schulz and K. H. Thiemann, Solid State Commun. 32, 783 (1979).

${ }^{38}$ A. Takagi, A. Nakamura, A. Yoshikaie, S. Yoshioka, S. Adachi, S. F. Chichibu, and T. Sota, J. Phys.: Condens. Matter 24, 415801 (2012).

${ }^{39} \mathrm{The}$ valence band ordering in $\mathrm{ZnO}$ is out of the scope of this paper. 